

\title{
Investigations into in situ Enterococcus faecalis biofilm removal by passive and active sodium hypochlorite irrigation delivered into the lateral canal of a simulated root canal model
}

\author{
S. A. Mohmmed ${ }^{1}$, M. E. Vianna ${ }^{2}$, M. R. Penny ${ }^{3}$, S. T. Hilton ${ }^{3}$, N. J. Mordan ${ }^{1}$, J. C.
} Knowles ${ }^{1}$

${ }^{1}$ Division of Biomaterials and Tissue Engineering, UCL Eastman Dental Institute, University College London, London, United Kingdom.

Postal address: 256 Grays Inn Road, London WC1X 8LD

E-mails: Saif.mohmmed.12@ucl.ac.uk

n.mordan@ucl.ac.uk

i.knowles@ucl.ac.uk

${ }^{2}$ School of Dentistry, College of Biomedical and Lifesciences, Department of Learning and Scholarship, Cardiff University, Cardiff, United Kingdom.

Postal address: Heath Park Campus, Cardiff CF14 4XY

Email:ViannaM@cardiff.ac.uk

${ }^{3}$ School of Pharmacy, Faculty of Life Sciences, University College London, London, United Kingdom.

Postal address: 406, 29-39 Brunswick Square, London WC1N 1AX

E-mail: matthew.penny@ucl.ac.uk

s.hilton@ucl.ac.uk

Corresponding author:

Jonathan Campbell Knowles

Division of Biomaterials and Tissue Engineering,

UCL Eastman Dental Institute,

256 Gray's Inn Road, London WC1X 8LD, UK.

Email: j.knowles@ucl.ac.uk

Running title: Biofilm removal by sodium hypochlorite.

Key words: Automated agitation; Enterococcus faecalis biofilm; lateral canal; manual agitation; sodium hypochlorite; 3D printing model. 


\section{Abstract}

\section{Aim}

To investigate in situ Enterococcus faecalis biofilm removal from the lateral canal of a simulated root canal system using passive or active irrigation protocols.

\section{Methodology}

Root canal models $(n=43)$ were manufactured from transparent resin materials using 3D-printing. Each canal was created with an $18 \mathrm{~mm}$ length, apical size 30, a .06 taper, and a lateral canal of $3 \mathrm{~mm}$ length, $0.3 \mathrm{~mm}$ diameter. Biofilms were grown on the lateral canal and apical $3 \mathrm{~mm}$ of the main canal for 10 days. Biofilm of three models was examined using SEM. The other forty models were divided to four groups $(\mathrm{n}=$ 10). The models were observed under a fluorescence microscope. Following $60 \mathrm{~s}$ of $9 \mathrm{~mL}$ of $2.5 \% \mathrm{NaOCl}$ irrigation using syringe and needle, the irrigant was either left stagnant in the canal or activated using gutta-percha, sonic or ultrasonic methods for $30 \mathrm{~s}$. Images were then captured every second using an external camera. The residual biofilm percentages were measured using image analysis software. The data were analyzed using generalized linear mixed models. A significance level of 0.05 was used throughout.

\section{Results}

The greatest level of biofilm removal was with ultrasonic agitation (66.76\%) followed by sonic (45.49\%), manual agitation (43.97\%), and passive irrigation groups (38.67\%) respectively. The differences were significant between the residual biofilm in the passive irrigation and both sonic \& ultrasonic groups $(P=0.001)$.

\section{Conclusion}


Agitation resulted in better penetration of the $2.5 \% \mathrm{NaOCl}$ into the lateral canal of an artificial root canal model. Ultrasonic agitation of $\mathrm{NaOCl}$ improved the removal of biofilm.

\section{Introduction}

It is widely acknowledged that complete eradication of biofilm by root canal treatment would be essential for preventing periodontitis, an inflammatory lesion around the dental root caused mainly by bacteria (Sjögren et al. 1997). Bacteria adhere to solid surfaces (in this case the root canal) where they divide and rapidly form biofilms (Costerton et al. 1999). Root canal treatment to remove biofilm includes instrumentation and irrigation. It has been reported that using instrumentation alone is not sufficient for complete elimination of bacteria from the root canal system (Nair et al. 2005). This inadequacy may be related in part to the irregular nature of the canal anatomy such that there are regions that do not come into contact with the instrument. Lateral canals that branch off the main root are examples of canal regions that are impossible to instrument and could harbour residual bacterial biofilms (Ricucci et al. 2009). Microorganisms that survive in the canal after treatment can cause secondary infections of the root canal system.

Enterococcus faecalis is an important pathogen in persistent root canal infections, often identified in teeth with post-treatment infection (Mejare 1974, Sedgley et al. 2004). Therefore, a final irrigation regimen performed after the completion of chemomechanical canal preparation may improve debridement in the non-instrumented part of the root canal system (Ballal et al. 2009). However, the debriding action of an irrigant may remain elusive when using a needle and syringe alone (Jiang et al. 2012). Agitation may be applied to aid the dispersal of the irrigant into the root canal system 
(Macedo et al. 2014). Agitation techniques for root canal irrigants include either manual agitation (Cecic et al. 1984, Druttman \& Stock 1989, Huang et al. 2008) or automated agitation (Cunningham et al. 1982, Sabins et al. 2003).

Manual agitation of the irrigant can be achieved by using a file (Bronnec et al. 2010) or a taped gutta-percha cone (Huang et al. 2008) placed in the instrumented canal and moved by the operator. Sonic and ultrasonic devices are examples of automated agitation (Sabins et al. 2003). These devices use a fine polymer or metal tip that is vibrated within the canal, with different frequencies depending on the instrument. Sonically activated instruments are driven with a frequency range of 1-6 kHz (Tronstad et al. 1985). In ultrasonic agitation, a tip oscillates at frequencies of 25 to $30 \mathrm{kHz}$ in a pattern of motion consisting of nodes and anti-nodes along its length (Weller et al. 1980).

Although recent evidence suggested that the efficacy of $\mathrm{NaOCl}$ could be enhanced by irrigant agitation (Layton et al. 2015, Mohmmed et al. 2016) or by increasing its concentration (Mohmmed et al. 2017), these studies focused on irrigation within simple straight root canals. However, the real-time monitoring of $\mathrm{NaOCl}$ efficacy in removing bacterial biofilm from a root canal with more complex anatomy has not been reported. Hence, more knowledge of biofilm- $\mathrm{NaOCl}$ interaction within the root canal system is crucial to improve the outcomes of the root canal treatment.

This study investigated the effect of different agitation techniques on the efficacy of $2.5 \% \mathrm{NaOCl}$ in eliminating the biofilm from the surface of the lateral canal using the residual biofilm, removal rate of biofilm, and the extent of destruction of the residual biofilm as outcome measures. 


\section{Materials and Methods}

The construction of the simulated root canal was performed according to Mohmmed et al. (2016) with the modification of a more complex root canal anatomy, ie. the presence of a lateral canal. The preparation of microbial strains, generation of biofilms, irrigation methods, data collection and analysis were based on (Mohmmed et al. 2016) and described in detail as follows.

\section{Construction of transparent root canal models with lateral canal and distribution to experimental groups}

The root canal models $(n=43)$ were created using a 3D printer (Formlabs Inc., Somerville, Massachusetts, USA) and, as each model comprised of two halves, a total of 86 half models were printed. The design of the model used herein consisted of a main canal of $18 \mathrm{~mm}$ length, apical size 30, a .06 taper, and a lateral canal of $3 \mathrm{~mm}$ length, $0.3 \mathrm{~mm}$ diameter located at $3 \mathrm{~mm}$ from the apical terminus (Figure 1). Three models were used to examine the biofilm generated on the surface of the root canal model. The other models were divided into four groups ( $\mathrm{n}=10$ per group) according to the type of irrigation protocol. In-group 1 (the passive irrigation group), no agitation was applied. In-group 2 (the manual agitation group), the irrigant was agitated using a gutta-percha cone (GP) (SybronEndo, Buffalo, New York, USA). In-group 3 (the sonic agitation group), the irrigant was agitated using the EndoActivator ${ }^{\circledR}$ device (Dentsply Tulsa Dental Specialties, Tulsa, Oklahoma, USA). In-group 4 (the ultrasonic agitation

group), the irrigant was agitated using a Satelec ${ }^{\circledR}$ P5 ultra-sonic device (Satelec, Acteon Inc, Laurel, New Jersy, USA). 


\section{Preparation of microbial strain and determination of the standard inoculum}

Biofilms were grown from a single bacterial strain (Enterococcus faecalis; ATCC 19433). The strain was supplied in the form of frozen stock in a brain-heart infusion broth (BHI) (Sigma-Aldrich, St. Louis, Montana, USA) and 30\% glycerol (Merck, Poole, UK) stored at $-70{ }^{\circ} \mathrm{C}$. The strain was thawed to $37^{\circ} \mathrm{C}$ for $10 \mathrm{~min}$ and swirled for $30 \mathrm{~s}$ using a Vortex mixer (IKA, Leighton Buzzard, UK) (Siqueira et al. 2002). After thawing, $100 \mu \mathrm{L}$ of the strain were taken and plated onto a BHI agar plate (Sigma-Aldrich) with $5 \%$ defibrinated horse blood (E\&O Laboratories, Bonnybridge, UK) and incubated at $37{ }^{\circ} \mathrm{C}$ in a $5 \% \mathrm{CO}_{2}$ incubator (LEEC, Nottingham, UK) for 24 h. Bacterial morphology and catalase activity were confirmed prior to the generation of the biofilms. For this, two colonies of the strain were removed separately using a sterile inoculating loop (VWR, Lutterworth, Leicester, UK), and catalase testing using $3 \% \mathrm{H}_{2} \mathrm{O}_{2}$ (SigmaAldrich Ltd, Poole, UK) and Gram staining (BD Ltd., Charlbury, UK), were performed. In addition, the identification of the strain was achieved by 16S rRNA gene sequencing and analysis.

A standard inoculum of $10^{8} \mathrm{CFU} / \mathrm{mL}$ concentration was used for the final biofilm production, which was adapted from a previous study (Al Shahrani et al. 2014). For this, six colonies were removed from the agar plate, placed into $20 \mathrm{~mL}$ of $\mathrm{BHI}$ broth with $5 \%$ defibrinated horse blood, and incubated at $37{ }^{\circ} \mathrm{C}$ in a $5 \% \mathrm{CO}_{2}$ incubator for $24 \mathrm{~h}$. BHI containing $E$. faecalis was adjusted to 0.5 absorbance at a wavelength of 600 nm using a spectrophotometer (NanoDrop ${ }^{\mathrm{TM}}$ Spectrophotometer ND-100, Wilmington, Delaware, USA) (AI Shahrani et al. 2014). Inoculum concentration was confirmed by determining the colony forming units per millilitre (CFUs $/ \mathrm{mL})$ using six ten-fold serial dilutions (Peters et al. 2001). This was performed by mixing aliquots of $100 \mu \mathrm{L}$ bacterial inoculum into $900 \mu \mathrm{L}$ of reduced transport fluid in $1.5 \mathrm{~mL}$ mini tubes 
(Sarstedt Ltd, Nümbrecht, Germany). From these dilutions, aliquots of $20 \mu \mathrm{L}$ were plated on $\mathrm{BHI}$ agar plates with $5 \%$ defibrinated horse blood and then incubated at 37 ${ }^{\circ} \mathrm{C}$ in the $5 \% \mathrm{CO}_{2}$ incubator for a period of $24 \mathrm{~h}$. The final concentration was $1.1 \times 10^{8}$ $\mathrm{CFU} / \mathrm{mL}$.

\section{Generation of single species biofilm (E. faecalis) on the surface of the apical 3 $\mathrm{mm}$ of the canal model}

The models halves were packed individually in packaging bags (Sterrad 100S, ASP ${ }^{\circledR}$, Irvine, California, USA) and then sterilised using gas plasma with hydrogen peroxide vapor (Sterrad 100S) for 50 min (Precautions \& Flush 2008). Each root canal model comprised of two halves but only one was used to grow a biofilm. This was so that, once assembled, the biofilm on the canal surface could be imaged through the half without a biofilm.

One $\mathrm{mL}$ of standard $E$. faecalis inoculum $\left(1.1 \times 10^{8} \mathrm{CFU} / \mathrm{mL}\right)$ was delivered into a sterilised $7 \mathrm{~mL}$ plastic bijou bottle (Sarstedt Ltd) that contained a sterilised half model. The apparatus was incubated at $37^{\circ} \mathrm{C}$ in a $5 \% \mathrm{CO} 2$ incubator for ten days. A sterile syringe (BD Plastipak ${ }^{T M}$, Franklin Lakes, NJ, USA) and a 21-gauge needle (BD Microlance $^{\mathrm{TM}}$, Franklin Lakes, NJ, USA) were used to immerse the $3 \mathrm{~mm}$ apical portion of the half model. Every three days, half of the inoculum that surrounded the model was discarded and replaced with fresh BHI broth (De-Deus et al. 2007). After incubation, three samples with biofilms were prepared for scanning electron microscopy and the remaining were assembled for irrigation treatment. 


\section{Re-apposition of the model halves}

Before reassembling the two model halves, a polyester seal film of $0.05 \mathrm{~mm}$ thickness (UnisealTM, Buckingham, UK) was positioned on the half coated with biofilm. Any part of the film that overhung the canal boundary was removed using a surgical blade (Swann-Morton, Owlerton, UK) without disturbing the biofilm. The two halves of the model were then held in position using four brass bolts (size $16 \mathrm{BA}$ ) and nuts (Clerkenwell Screws, London, UK).

\section{Irrigation experiments}

In order to image the biofilm and it's removal, the model halves with biofilms were removed from the plastic bottle and prepared for staining with a crystal violet (CV) stain (Merck, Darmstadt, Germany). CV was used as it stained the biofilm such that it could be distinguished from the model and did not bleach with the $\mathrm{NaOCl}$. Each model half was placed on a microscope slide and rinsed with distilled water (Roebuck, London, UK) to remove loosely attached cells. Using a pipette (Alpha Laboratories Ltd, Eastleigh, UK), $2 \mu \mathrm{L}$ of CV stain were applied to the part of the canal half where the biofilm had been generated $(3 \mathrm{~mm})$ and left for 1 minute for staining. It was subsequently washed with distilled water (Izano et al. 2007)

In all groups, sodium hypochlorite $(\mathrm{NaOCl})\left(\right.$ Teepo $^{\circledR}$ bleach, Teepol products, Egham, UK) of $2.5 \%$ available chlorine and $12.8 \mathrm{pH}$ was used as irrigating solution. $9 \mathrm{~mL}$ of the $\mathrm{NaOCl}$ was delivered using a $10 \mathrm{~mL}$ syringe (Plastipak) with a 27 -gauge side-cut open-ended needle (Monoject, Sherwood Medical, St. Louis, Missouri, USA). The needle was inserted into the canal just coronal to the organic film or biofilm. The port opening of the needle always faced the model half containing the organic film or biofilm. The syringe was attached to a programmable precision syringe pump (NE- 
1010; New Era Pump Systems, Wantagh, New York, USA) in order to deliver the irrigant at a flow rate of $0.15 \mathrm{~mL} \mathrm{~s}^{-1}$. For each canal, a total of $9 \mathrm{~mL}$ of irrigant was delivered over a period of $1 \mathrm{~min}$. In group 1, following the $60 \mathrm{~s}$ irrigation using a syringe and needle, the irrigant was kept stagnant (passive irrigation) in the canal for $30 \mathrm{~s}$ in the other groups (2-4), the irrigating solution was agitated using manual (Group 2) , sonic (Group 3) and ultrasonic methods (Group 4) .

In the manual agitation group, the irrigant was delivered as in the previous group. Following that, a gutta-percha cone with an apical size 30 and 0.02 taper was placed $2 \mathrm{~mm}$ coronal to the canal terminus which was used to agitate the irrigant in the root canal system with a push-pull amplitude of approximately 3-5 $\mathrm{mm}$ at a frequency of 50 strokes per 30 s (Huang et al. 2008). A new GP cone was used with each canal model.

For the sonic agitation group, the irrigant was delivered as described in group 1. Following that, the agitation was carried out using an EndoActivator ${ }^{\circledR}$ device by placing the polymer tip of an EndoActivator ${ }^{\circledR}$ device with size 25 and 0.04 taper at $2 \mathrm{~mm}$ from the canal terminus, and then the agitation was continued for $30 \mathrm{~s}$ with a high powersetting (Ruddle 2007). A new tip was used with each canal model.

For the ultrasonic agitation group, the irrigant was delivered as in the previous group. Following that, the agitation was carried out by placing a stainless steel instrument, size and taper 20/02 (IrriSafe; Satelec Acteon, Merignac, France), of a Satelec ${ }^{\circledR}$ P5 Newtron piezon unit at $2 \mathrm{~mm}$ from the canal terminus, then the agitation was continued for $30 \mathrm{~s}$. The file was energized at power setting 7 as recommended by the manufacturer. A new instrument was used with each canal model. 
Following irrigation protocols, the residual $\mathrm{NaOCl}$ on the model surface was immediately neutralised by immersing the models in $2 \mathrm{~mL}$ of $5 \%$ sodium thiosulphate solution (Sigma-Aldrich Co Ltd., Gillingham, UK) for 5 minutes (Hegde et al. 2012).

Three models from each group were examined for residual biofilm using SEM. The residual biofilm on the canal surface was imaged at 3,2 , and $1 \mathrm{~mm}$ from the canal terminus. Three models from each group were examined to assess the effect of $2.5 \%$ $\mathrm{NaOCl}$ irrigant on the residual surface biofilm.

\section{Recording of biofilm removal by the irrigant}

The rate of film or biofilm removal was recorded using a high-resolution CCD camera (QICAM Mono Fast 1479, Toronto, ON, Canada). The camera was connected to a fluorescent microscope (Leica DMIRB, Leica Microsystems UK, Mlton Keynes, UK) and the samples imaged with a $2.5 \times$ magnification lens. During the time-lapse recording of interactions between the irrigant and the organic film or biofilm, both fluorescing (red filter) and non-fluorescing (intensity of $2.5 \mathrm{~W} / \mathrm{m}^{2}$ ) light was used to achieve a better resolution (Figure 2).

\section{Image analysis}

The video-captured recording was separated into ninety images according to each second of footage using Image $\mathrm{J} 1.4$ and micro-imaging software 1.4 (Media Cybernetics Inc., Rockville, MD, USA). The images were analysed using Image-pro Plus 4.5 and ipWin4 software (MediaCybernetics ${ }^{\circledR}$, Silver Spring, Maryland, USA). Canal surface coverage by residual organic film or biofilm present after every second of irrigation $(0.15 \mathrm{~mL})$ was quantified. 


\section{Preparation of the samples for Scanning Electron Microscopy}

The biofilm on the surface of three untreated root canal models and three models from each treatment group were observed using scanning electron microscopy (SEM) (FEI XL30 FEG SEM, FEI, Eindhoven, Netherlands). For this, samples were fixed in $3 \%$ glutaraldehyde (Agar Scientific, Stansted, UK) in $0.1 \mathrm{M}$ sodium cacodylate buffer (Agar Scientific,) at $4{ }^{\circ} \mathrm{C}$ overnight. Then, they were dehydrated in a graded series of ethanol (50, 70, 90, and 100\%) (Sigma-Aldrich Co Ltd.,), placed in hexamethyldisilazane (Agar Scientific,) for $5 \mathrm{~min}$ and air-dried. Samples were mounted onto aluminium pin stubs (Agar Scientific,), and sputter coated with gold/palladium (Polaron E5000, QUORUM Technology, Laughton, UK) before examination using SEM. The appearance of the remaining biofilm was assessed as was the morphology of the bacteria.

\section{Data analyses}

The residual biofilm (\%) on the surface of the root canal model with a lateral canal anatomy at each second of $90 \mathrm{~s}$ irrigation with passive and active $2.5 \% \mathrm{NaOCl}$ irrigant was analysed using line plots. An assumption concerning a normal distribution of data for the residual biofilm was checked using a visual inspection of the box and whisker plots. The data were normally distributed and therefore the generalised linear mixed models, followed by Dunnett post-hoc comparisons were performed to compare their distributions in the four experimental groups. A similar analysis was performed to analyse the effects of irrigant agitation duration (time) and experimental group (passive or manual, sonic, and ultrasonic active irrigation) on the percentage of residual biofilm covering the lateral canal surface area. A significance level of 0.05 was used throughout. The data were analysed by SPSS (BM Corp. Released 2013. IBM SPSS Statistics for Windows, Version 22.0. Armonk, New York, USA). 


\section{Results}

The greatest removal of $E$. faecalis biofilm was associated with the ultrasonic agitation protocol followed by sonic agitation, manual agitation, and passive irrigation (control) protocols respectively (Figure 3 ).

The mean (95\% Confidence interval) percentages of the lateral canal surface area coverage with residual bacterial biofilm against duration of irrigation(s) are presented in Figure 4. The data showed that the greatest removal was associated with the ultrasonic agitation group (66.76\%) followed by sonic agitation (45.49\%), manual agitation (43.97\%), and passive irrigation groups (control) (38.67\%) respectively.

The results from the linear mixed model (Table 1) indicated that there was a significant difference between the residual biofilm on the lateral canal surface area in the passive irrigation group and the automated groups (sonic \& ultrasonic) $(P=0.001)$. Amongst the agitation groups, strong evidence of less residual biofilm was found in the ultrasonic agitation group than those in the sonic and manual agitation groups $(P=$ $0.011)$.

Another important finding (Table 2) was that the interval of irrigant agitation interestingly had an influence on the amount of biofilm removed. The amount of biofilm removed using passive irrigation group was significantly less $[0.51 \% / \mathrm{s} ;( \pm 0.08)$, $1.01 \% / \mathrm{s} ;( \pm 0.08)]$ than the amount of biofilm removed using sonic, and ultrasonic agitation groups respectively $(P=0.001)$. For the agitation groups, the amount of biofilm removed using the ultrasonic agitation group was significantly more $[0.07 \% / \mathrm{s}$; $( \pm 0.06), 0.49 \% / \mathrm{s} ;( \pm 0.06)]$ than that using the manual and sonic agitation group respectively $(P=0.001)$.

SEM images of the biofilm on the surface of the lateral canal models before and after irrigation are presented in Figure 5. 
Taking the biofilm structure of the untreated model into account, SEM images (Fig. 5ai) showed cocci morphology of the bacteria cell. Bacterial cells were often gathered in colonies, and held together by a matrix of extracellular polymeric substance. Complete encapsulation of bacterial cells by the matrix could be observed.

The influences of $2.5 \% \mathrm{NaOCl}$ irrigation on biofilm at the $3 \mathrm{~mm}$ level from the canal terminus are presented in Figure 5 (aii, aiii, aiv). Although SEM images of passive irrigation (Fig. 5aii) and manual agitation (Fig. 5aiii) groups showed residual biofilm with obvious ESP destruction and a damaged cell membrane; some bacteria cells appeared flawless. Entire biofilm elimination was associated with automated groups (Fig. 5aiv).

At the $2 \mathrm{~mm}$ level, reduction in removal and destruction effect were evident in the passive irrigation (Fig. 5bi) and manual (Fig. 5ci) groups, and communities of bacterial cells held by EPS matrix were noted. This effect was more distinct in the former group. Regarding the automated groups, the greatest biofilm deformation and removal was associated with the ultrasonic group (Fig. 5ei) followed by the sonic group (Fig. 5di).

At $1 \mathrm{~mm}$ from the canal terminus, both passive irrigation (Fig. 5bii) and manual (Fig. 5cii) groups had no effect and this pattern was reflected in the intact form and structure of the biofilm. The destruction of biofilm by $\mathrm{NaOCl}$ was noticed in the sonic (Fig. 5 dii) and ultrasonic (Fig. 5eii) groups. This effect was superior in the latter group. However, unharmed bacterial cells that are enclosed in an extracellular polymeric substance was identified.

\section{Discussion}

This study set out with the aim of comparing the impact of passive and active irrigation protocols (manual, sonic, and ultrasonic agitation) and time of irrigation on the efficacy 
of $2.5 \% \mathrm{NaOCl}$ irrigant in biofilm removal from the wall of a simulated lateral canal of the root canal system. The results did not show any significant increase in the efficacy of $\mathrm{NaOCl}$ during manual agitation. Although a greater removal and eradication effect of $\mathrm{NaOCl}$ on the $E$. faecalis biofilm was associated with the ultrasonic activation group, it was not enough for complete biofilm removal and dissolution from the lateral canal anatomy.

In the present study, all laboratory models were made of synthetic transparent materials. The surface and composition of such materials differ from that of root canal dentine. The porous nature of dentine (due to dentinal tubules) may act differently from a solid plastic material. A laboratory study that uses ex vivo (extracted teeth) to test the antimicrobial action of irrigants would be more relevant in terms of reflecting the clinical situation. Yet, tooth dentine is optically opaque and any internal structures are concealed, which makes them inaccessible for the direct visualisation needed to assess the antibacterial action of an irrigant during the process of irrigation. In addition, the ethical issues and difficulties in patient recruitment may limit the ability of achieving adequate sample sizes (Spratt et al. 2001). In this regard, the models advocated in this study have the advantage that the transparent canal model allows for a direct investigation in a time dependent way, into the removal action of the test targets (biofilm, simulant biofilms) by $\mathrm{NaOCl}$ irrigant. In addition, the use of the 3D printing technique with stereolithography materials allows the generation of multiple samples with the same anatomical features and much finer resolution (Melchels et al. 2010). Major criticism of this model is that the attachment of the biofilm in dentine may differ from that on a synthetic material of the model used here. Nevertheless, previous investigations, which examined the potential of the 3D printing materials for development of suitable in vitro biofilm models illustrated that the model material 
allowed for attachment and growth of E. faecalis biofilm on their surface to a similar extent to that of dentine (Mohmmed et al. 2016).

In this study, the root canal model was created with an apical size 30, .06 taper because it has been suggested that the minimum apical size necessary to deliver the irrigant to the canal terminus is size 30 (Khademi et al. 2006). A side cut 27-gauge endodontic needle was chosen, as it is commonly used in clinical practice, and to avoid the greater pressure required to deliver the irrigant at a rate of $9 \mathrm{~mL}$ per minute, as is the case when using a flat ended 30-gauge needle (Shen et al. 2010). A total of $9 \mathrm{~mL}$ of $\mathrm{NaOCl}$ were used during syringe irrigation protocol as it has been reported that 9 $\mathrm{mL}$ were sufficient to remove stained collagen simulating biofilm from the root canal system (Huang et al. 2008). The volume of $9 \mathrm{~mL}$ per minute $\left(0.15 \mathrm{~mL} \mathrm{~s}^{-1}\right)$ irrigant was selected as an attempt to improve the solution penetration (Bronnec et al. 2010). Furthermore, this rate falls within the range of $0.01-1.01 \mathrm{~mL} \mathrm{~s}^{-1}$ reported in previous studies to be used in clinical practice (Boutsioukis et al. 2007). One criticism may be generated about the high flow rate that may increase both apical pressure and irrigant extrusion (Park et al. 2013); however, it has been argued that the healthy condition of the periapical tissue creates a barrier against apical extrusion (Salzgeber \& Brilliant 1977).

The diameter of the lateral canal of the root canal model was $0.3 \mathrm{~mm}(300 \mu \mathrm{m})$. This may be considered as a limitation as it lies beyond the range of the lateral canals (10 - $200 \mu \mathrm{m}$ ) reported in previous studies using scanning electron microscope (Dammaschke et al. 2004) and microcomputer tomography (Al-Jadaa et al. 2009) of human teeth. However, this width was selected, as it was adequate for recording the in-situ removal of the bacterial biofilm. In addition, based on previous observations on the printing of lateral canal models with a smaller diameter, the inner surface of the 
canal was incompletely polymerised. Furthermore, the lateral canal of diameter 250 $\mu \mathrm{m}$, which is larger than the abovementioned range, was used in a previous study to investigate the removal of simulated biofilms from the lateral canals (Macedo et al. 2014).

A total of ten days was selected for biofilm growth as it has been confirmed that this period allowed microbial colonization and developed biofilm models (Sena et al. 2006). The relevant biofilm model allowed for the controlled investigation and comparison of the antimicrobial protocols (Halford et al. 2012). Antimicrobial susceptibility of generated biofilms over time has been explored intensively. For instance, Wang et al. (2012) reported that young biofilm was more sensitive to intracanal medicaments, and bacteria were more easily killed than in an old biofilm. It has been argued that biofilms become increasingly difficult to eliminate by antibacterial agents between 2 and 3 weeks (Stojicic et al. 2013). However, another study suggested the biofilm resistance is inherent and it is possible to generate mature wild bacterial biofilm (Pseudomonas aeruginosa) after 5 days incubation (Klausen et al. 2003).

In the present study, an inverted fluorescent microscope was selected to observe and record biofilm removal by $\mathrm{NaOCl}$. The main advantage of this microscope was that it allowed direct imaging of the biofilm removal without the need for sample fixation. However, high resolution imaging proved difficult because of the steeply curved sides of the canal walls that resulted in a lot of out-of-focus data and increased reflected light. Furthermore, it was not possible to assess single bacterial cell destruction in the biofilm because a low magnification $2.5 x$ lens was needed in order to observe the whole apical canal in a single image. In this regard, residual biofilms were examined using SEM to assess the extent of destruction of biofilm structure. 
The use of crystal violet stain to render the biofilm visible under the microscope provoked an issue, because the stain might have affected the oxidative capability of $\mathrm{NaOCl}$. For this, experiments were performed to examine the effect of $\mathrm{CV}$ stain on the oxidative capacity and capability of $\mathrm{NaOCl}$. The results showed that $\mathrm{CV}$, which displayed a fluorescent capacity, had a neutral effect on $\mathrm{NaOCl}$. This was interpreted by the evaluation of the available chlorine and $\mathrm{pH}$ of $\mathrm{NaOCl}$ before and after the addition of CV. This result may be attributed to the alkaline property of the stain, or due to its concentration, which was not high enough to affect the oxidative capacity of $\mathrm{NaOCl}$. The experiments were performed in triplicate.

Image analysis software (Image-Pro Plus) has been used to analyse the images from the fluorescent microscope. This software has been adopted in other studies in order to analyse images (Huang et al. 2008, McGill et al. 2008). One criticism that can be made in relation to all image-analysis techniques is that the areas measured are to some extent subjectively chosen by the examiner. In order to reduce this limitation, inter- and intra-examiner assessments were carried out. A semi-automatic approach to measuring the biofilms was applied and imaging software was used to manually draw the template of the root canal outline and quantify the biofilm. The same template was used to obtain and calculate the biofilm area after washing, without further interference of the operator.

Although the method of quantifying the biofilm from the root canal wall revealed marked results, a single assessor performed the measurements and therefore there was a possibility of bias. In order to reduce this, a methodology was agreed using a standard protocol for outlining the root canal and for setting the threshold of the stain to be measured. The principal assessor and another observer who was experienced in using image analysis software measured $10 \%$ of the images and this was repeated 
until sufficient inter-observer agreement was achieved (Hartmann \& Wood 1990). Another attempt to reduce bias was attained by assessment of the intra-observer reliability. This was performed by recording ten replicate measurements of the residual biofilm in each group at specific intervals (every $10 \mathrm{~s}$ of the $90 \mathrm{~s}$ irrigation) and comparing the values taken. This comparison showed good agreement between the measurements (Koppe et al. 2009). This semi-automatic method provided operatorindependent quantitative results.

The biofilms on the surface of the test materials were observed using SEM, which provided information about the structure and components of the single species biofilm formed on the test materials. However, the study did not attempt to measure the thickness of the biofilm. The reason for this is related to the effect of the dehydration procedure during SEM sample preparation, which may cause shrinkage of the biofilm, affecting it's thickness (Paz et al. 2015).

This study did not include the use of neutral irrigation solutions (saline, water) to clarify whether the biofilm removal was a result of the chemical activity of $\mathrm{NaOCl}$ or the physical characteristic of the agitation protocols used in the study. The current research was specifically designed to evaluate the effect of different agitation protocols on the ability of $\mathrm{NaOCI}$ to remove and disrupt $E$. faecalis biofilm in comparison with a passive irrigation protocol. It has been reported in our previous study that the residual biofilms after irrigation protocol without agitation using $5.25 \% \mathrm{NaOCl}$ and $2.5 \% \mathrm{NaOCl}$ were less than that using water $(P=0.001)$. However, it would be interesting to repeat that study using different agitation methods.

One result which emerged from the statistical analysis was that $\mathrm{NaOCl}$ should to be in direct contact with the $E$. faecalis biofilm to perform total removal and destruction of 
the bacterial cell (Moorer \& Wesselink 1982). This was achieved in all groups at the 3 $\mathrm{mm}$ level from the lateral canal terminus, as the port opening of the needle was facing the lateral canal, which may yield a jet with high velocity fluid flow (Boutsioukis et al. 2010, Verhaagen et al. 2012).

The agitation of the $\mathrm{NaOCl}$ could enhance a lateral flow component, and improve irrigant penetration into the side canal (Castelo-Baz et al. 2012). However, no complete eradication of biofilm was evident in the passive and manual agitation groups. The possible explanation for this might be that the rate of irrigant refreshment as the irrigant diffused was decreased (van der Sluis et al. 2010). As the irrigation procedure continued, the irrigant penetration into the terminus of the lateral canals was enhanced with automated groups (sonic and ultrasonic). These results may be related to the acoustic streaming and cavitation effects that were created by the tip oscillation of the sonic and ultrasonic device within the main root canal (van der Sluis et al. 2005). Nevertheless, $\mathrm{NaOCl}$ efficacy was insufficient for complete removal of the residual biofilm. This could be due to fact that the effective diffusion of $\mathrm{NaOCl}$ was restricted to the top layers of the biofilm (Renslow et al. 2010). Another possible explanation for this is the rapid consumption of $\mathrm{OCl}^{-}$ions of $\mathrm{NaOCl}$ during its reaction with biofilm (Moorer \& Wesselink 1982). The efficacy of $\mathrm{NaOCl}$ was reduced at $1 \mathrm{~mm}$ from the lateral canal terminus in all irrigation groups. This observation could be attributed to the reduction in both fluid convection (Verhaagen et al. 2014) and irrigant replacement (Wang et al. 2014).

It is important to consider the effect of increasing $\mathrm{NaOCl}$ concentration on the amount of biofilm removal as it has been reported that tissue dissolution of $\mathrm{NaOCl}$ increased with a higher concentration of the $\mathrm{NaOCl}$ solution (Moorer \& Wesselink 1982 , Haapasalo et al. 2014). Furthermore, it has been argued that agitation improved the 
antimicrobial efficacy of $5.25 \% \mathrm{NaOCl}$ when compared with $2.5 \% \mathrm{NaOCl}$ (Sena et al. 2006). Although these studies provided information about the efficacy of irrigation, their results were based upon a technique of immersing the sample into an irrigant. In such conditions, the efficacy of irrigation was related to its chemical action as well as diffusion without interference of the canal confinement, which may reduce the mixing and replacement of irrigant (Verhaagen et al. 2012). In this regard, Mohmmed et al. (2017) reported that a clear improvement in biofilm removal was achieved when $5.25 \%$ of $\mathrm{NaOCl}$ was used instead of $2.5 \%$, but no complete biofilm removal was detected. This suggest that the flow of $\mathrm{NaOCl}$ to the full extent of the root canal system by frequent refreshment/agitation cycles may be more important for obtaining maximal removal effect than increasing its concentration.

The findings are in agreement with de Gregorio et al. (2009) findings, who showed that the efficacy of the automated groups (sonic \& ultrasonic) was greater than that of the passive irrigation group. However, the findings of the current study do not support the abovementioned study, which reported that there was no difference between the sonic and ultrasonic agitation groups. This inconsistency may be due to the structure of biofilm exhibiting resistance to antimicrobial agents (Roberts \& Mullany 2010) when compared to the contrast media used in the de Gregorio et al. study. 
The presence of a lateral canal influenced the dynamic of fluids, resulting in lower percentage of $E$. faecalis biofilm removal in the groups that received active irrigation when compared to single canal anatomy as reported by Mohmmed et al. (2016). The canal complexicity reduced the cleaning efficiency by $23 \%$ in the ultrasonic agitation, $35 \%$ in the sonic, and $37 \%$ in the manual agitation groups. Hence, increasing canal complexity results in changes in the mechanics of fluids and future studies should consider increasing the canal complexity and microbial variety, considering multispecies biofilms.

\section{Conclusion}

The removal effect of $\mathrm{NaOCl}$ on bacterial biofilm was limited to the $3 \mathrm{~mm}$ level from the lateral canal terminus. The agitation of $\mathrm{NaOCl}$ resulted in better penetration of the irrigant into the lateral canals. Ultrasonic agitation of $\mathrm{NaOCl}$ improved the removal of biofilm.

\section{Acknowledgements}

The authors declare that there is no conflict of interest. The authors acknowledge that this study is based on ideas and concepts originated, developed and under investigation by Professor Kishor Gulabivala and Yuan Ng, strands of which have been presented at conferences and as Masters Dissertations. This work was partially supported by the Iraqi Ministry of Higher Education through a PhD Scholarship (\# 1031). 


\section{References}

Al-Jadaa A, Paqué F, Attin T, Zehnder M (2009) Necrotic pulp tissue dissolution by passive ultrasonic irrigation in simulated accessory canals: impact of canal location and angulation. International Endodontic Journal 42, 59-65.

Al Shahrani M, DiVito E, Hughes CV, Nathanson D, Huang GT-J (2014) Enhanced Removal of Enterococcus faecalis Biofilms in the Root Canal Using Sodium Hypochlorite Plus Photon-Induced Photoacoustic Streaming: An In Vitro Study. Photomedicine and Laser Surgery 32, 260-6.

Ballal NV, Kandian S, Mala K, Bhat KS, Acharya S (2009) Comparison of the efficacy of maleic acid and ethylenediaminetetraacetic acid in smear layer removal from instrumented human root canal: a scanning electron microscopic study. Journal of Endodontics 35, 1573-6.

Boutsioukis C, Lambrianidis T, Kastrinakis E, Bekiaroglou P (2007) Measurement of pressure and flow rates during irrigation of a root canal ex vivo with three endodontic needles. International Endodontic Journal 40, 504-13.

Boutsioukis C, Verhaagen B, Versluis M, Kastrinakis E, van der Sluis LW (2010) Irrigant flow in the root canal: experimental validation of an unsteady Computational Fluid Dynamics model using high-speed imaging. International Endodontic Journal 43, 393-403.

Bronnec F, Bouillaguet S, Machtou P (2010) Ex vivo assessment of irrigant penetration and renewal during the final irrigation regimen. International Endodontic Journal 43, 663-72.

Castelo-Baz P, Martín-Biedma B, Cantatore G et al. (2012) In vitro comparison of passive and continuous ultrasonic irrigation in simulated lateral canals of extracted teeth. Journal of Endodontics 38, 688-91. 
Cecic PA, Peters DD, Grower MF (1984) The comparative efficiency of final endodontic cleansing procedures in removing a radioactive albumin from root canal systems. Oral Surgery, Oral Medicine, Oral Pathology 58, 336-42.

Costerton JW, Stewart PS, Greenberg E (1999) Bacterial biofilms: a common cause of persistent infections. Science 284, 1318-22.

Cunningham WT, Martin H, Forrest WR (1982) Evaluation of root canal debridement by the endosonic ultrasonic synergistic system. Oral Surgery, Oral Medicine, Oral Pathology 53, 401-4.

Dammaschke T, Witt M, Ott K, Schäfer E (2004) Scanning electron microscopic investigation of incidence, location, and size of accessory foramina in primary and permanent molars. Quintessence International 35, 699-705.

De-Deus G, Brandão M, Fidel R, Fidel S (2007) The sealing ability of GuttaFlow ${ }^{\mathrm{TM}}$ in oval-shaped canals: an ex vivo study using a polymicrobial leakage model. International Endodontic Journal 40, 794-9.

de Gregorio C, Estevez R, Cisneros R, Heilborn C, Cohenca N (2009) Effect of EDTA, Sonic, and Ultrasonic Activation on the Penetration of Sodium Hypochlorite into Simulated Lateral Canals: An In Vitro Study. Journal of Endodontics 35, 891-5.

Druttman A, Stock C (1989) An in vitro comparison of ultrasonic and conventional methods of irrigant replacement. International Endodontic Journal 22, 174-8.

Haapasalo M, Wang Z, Shen Y, Curtis A, Patel P, Khakpour M (2014) Tissue Dissolution by a Novel Multisonic Ultracleaning System and Sodium Hypochlorite. Journal of Endodontics 40, 1178-81. 
Halford A, Ohl C-D, Azarpazhooh A, Basrani B, Friedman S, Kishen A (2012) Synergistic Effect of Microbubble Emulsion and Sonic or Ultrasonic Agitation on Endodontic Biofilm in Vitro. Journal of Endodontics 38, 1530-4.

Hartmann DP, Wood DD (1990) Observational methods. International handbook of behavior modification and therapy, 2 edn; pp. 107-138. Plenum Press, New York: Springer/USA.

Hegde J, Bashetty K, Krishnakumar UG (2012) Quantity of sodium thiosulfate required to neutralize various concentrations of sodium hypochlorite. Asian Journal of Pharmaceutical and Health Sciences 2, 390-3.

Huang TY, Gulabivala K, Ng YL (2008) A bio-molecular film ex-vivo model to evaluate the influence of canal dimensions and irrigation variables on the efficacy of irrigation. International Endodontic Journal 41, 60-71.

Izano EA, Wang H, Ragunath C, Ramasubbu N, Kaplan JB (2007) Detachment and killing of Aggregatibacter actinomycetemcomitans biofilms by dispersin B and SDS. Journal of Dental Research 86, 618-22.

Jiang L-M, Lak B, Eijsvogels LM, Wesselink P, van der Sluis LW (2012) Comparison of the cleaning efficacy of different final irrigation techniques. Journal of Endodontics 38, 838-41.

Khademi A, Yazdizadeh M, Feizianfard M (2006) Determination of the minimum instrumentation size for penetration of irrigants to the apical third of root canal systems. Journal of Endodontics 32, 417-20.

Klausen M, Heydorn A, Ragas P et al. (2003) Biofilm formation by Pseudomonas aeruginosa wild type, flagella and type IV pili mutants. Molecular Microbiology 48, $1511-24$. 
Koppe T, Meyer G, Alt K (2009) Comparative dental morphology. Preface. Frontiers of Oral Biology 13, XI.

Layton G, Wu W-I, Selvaganapathy PR, Friedman S, Kishen A (2015) Fluid Dynamics and Biofilm Removal Generated by Syringe-delivered and 2 Ultrasonic-assisted Irrigation Methods: A Novel Experimental Approach. Journal of Endodontics 41, 884-9.

Macedo R, Robinson J, Verhaagen B et al. (2014) A novel methodology providing insights into removal of biofilm-mimicking hydrogel from lateral morphological features of the root canal during irrigation procedures. International Endodontic Journal 47, 1040-51.

McGill S, Gulabivala K, Mordan N, Ng YL (2008) The efficacy of dynamic irrigation using a commercially available system (RinsEndo®) determined by removal of a collagen 'bio-molecular film'from an ex vivo model. International Endodontic Journal 41, 602-8.

Mejare B (1974) Streptococcus faecalis and Streptococcus faecium in infected dental root canals at filling and their susceptibility to azidocillin and some comparable antibiotics. Odontologisk Revy 26, 193-204.

Melchels FP, Feijen J, Grijpma DW (2010) A review on stereolithography and its applications in biomedical engineering. Biomaterials 31, 6121-30.

Mohmmed SA, Vianna ME, Hilton ST, Boniface DR, Ng YL, Knowles JC (2016) Investigation to test potential stereolithography materials for development of an in vitro root canal model. Microscopy Research and Technique 80, 202-10. Mohmmed SA, Vianna ME, Penny MR, Hilton ST, Knowles JC (2017) The effect of sodium hypochlorite concentration and irrigation needle extension on biofilm 
removal from a simulated root canal model. Australian Endodontic Journal. doi:10.1111/aej.12203.

Mohmmed SA, Vianna ME, Penny MR, Hilton ST, Mordan N, Knowles JC (2016) A novel experimental approach to investigate the effect of different agitation methods using sodium hypochlorite as an irrigant on the rate of bacterial biofilm removal from the wall of a simulated root canal model. Dental Materials 32, 1289-300.

Moorer W, Wesselink P (1982) Factors promoting the tissue dissolving capability of sodium hypochlorite. International Endodontic Journal 15, 187-96.

Nair P, Henry S, Cano V, Vera J (2005) Microbial status of apical root canal system of human mandibular first molars with primary apical periodontitis after "onevisit" endodontic treatment. Oral Surgery, Oral Medicine, Oral Pathology, Oral Radiology, and Endodontology 99, 231-52.

Park E, Shen Y, Khakpour M, Haapasalo M (2013) Apical pressure and extent of irrigant flow beyond the needle tip during positive-pressure irrigation in an in vitro root canal model. Journal of Endodontics 39, 511-5.

Paz LECd, Sluis L, Boutsioukis C et al. (2015) The root canal biofilm, Verlag Berlin Heidelberg: Springer.

Peters LB, Wesselink PR, Buijs JF, van Winkelhoff AJ (2001) Viable Bacteria in Root Dentinal Tubules of Teeth with Apical Periodontitis. Journal of Endodontics 27, 76-81.

Precautions MSB, Flush AC (2008) Guideline for Disinfection and Sterilization in Healthcare Facilities, 2008. [WWW document]. http://www.cdc.gov/hicpac/pdf/guidelines/Disinfection-Nov 2008.pdf URL| [accessed on 08.07.2015]. 
Renslow RS, Majors PD, McLean JS, Fredrickson JK, Ahmed B, Beyenal H (2010) In situ effective diffusion coefficient profiles in live biofilms using pulsed-field gradient nuclear magnetic resonance. Biotechnology and Bioengineering 106, 928-37.

Ricucci D, Siqueira JF, Bate AL, Ford TRP (2009) Histologic investigation of root canal-treated teeth with apical periodontitis: a retrospective study from twenty-four patients. Journal of Endodontics 35, 493-502.

Roberts AP, Mullany P (2010) Oral biofilms: a reservoir of transferable, bacterial, antimicrobial resistance. Expert Review of Anti-Infective Therapy 8, 1441-50.

Ruddle CJ (2007) Hydrodynamic disinfection. Dentistry Today 11, 1-9.

Sabins RA, Johnson JD, Hellstein JW (2003) A comparison of the cleaning efficacy of short-term sonic and ultrasonic passive irrigation after hand instrumentation in molar root canals. Journal of Endodontics 29, 674-8.

Salzgeber RM, Brilliant JD (1977) An in vivo evaluation of the penetration of an irrigating solution in root canals. Journal of Endodontics 3, 394-8.

Sedgley C, Lennan S, Clewell D (2004) Prevalence, phenotype and genotype of oral enterococci. Oral Microbiology and Immunology 19, 95-101.

Sena N, Gomes B, Vianna M et al. (2006) In vitro antimicrobial activity of sodium hypochlorite and chlorhexidine against selected single-species biofilms. International Endodontic Journal 39, 878-85.

Shen Y, Gao Y, Qian W et al. (2010) Three-dimensional numeric simulation of root canal irrigant flow with different irrigation needles. Journal of Endodontics 36, 884-9. 
Siqueira JF, Rôças IN, Lopes HP (2002) Patterns of microbial colonization in primary root canal infections. Oral Surgery, Oral Medicine, Oral Pathology, Oral Radiology, and Endodontology 93, 174-8.

Sjögren U, Figdor D, Persson S, Sundqvist G (1997) Influence of infection at the time of root filling on the outcome of endodontic treatment of teeth with apical periodontitis. International Endodontic Journal 30, 297-306.

Spratt D, Pratten J, Wilson M, Gulabivala K (2001) An in vitro evaluation of the antimicrobial efficacy of irrigants on biofilms of root canal isolates. International Endodontic Journal 34, 300-7.

Stojicic S, Shen Y, Haapasalo M (2013) Effect of the source of biofilm bacteria, level of biofilm maturation, and type of disinfecting agent on the susceptibility of biofilm bacteria to antibacterial agents. Journal of Endodontics 39, 473-7.

Tronstad L, Barnett F, Schwartzben L, Frasca P (1985) Effectiveness and safety of a sonic vibratory endodontic instrument. Dental Traumatology 1, 69-76.

Van der Sluis L, Wu MK, Wesselink P (2005) The efficacy of ultrasonic irrigation to remove artificially placed dentine debris from human root canals prepared using instruments of varying taper. International Endodontic Journal 38, 7648.

van der Sluis LW, Vogels MP, Verhaagen B, Macedo R, Wesselink PR (2010) Study on the influence of refreshment/activation cycles and irrigants on mechanical cleaning efficiency during ultrasonic activation of the irrigant. Journal of Endodontics 36, 737-40.

Verhaagen B, Boutsioukis C, Heijnen G, Van der Sluis L, Versluis M (2012) Role of the confinement of a root canal on jet impingement during endodontic irrigation. Experiments in fluids 53, 1841-53. 
Verhaagen B, Boutsioukis C, Sleutel C, Kastrinakis E, Van der Sluis L, Versluis M (2014) Irrigant transport into dental microchannels. Microfluidics and nanofluidics 16, 1165-77.

Wang Y, da Silva Domingues JF, Subbiahdoss G, van der Mei HC, Busscher HJ, Libera M (2014) Conditions of lateral surface confinement that promote tissuecell integration and inhibit biofilm growth. Biomaterials 35, 5446-52.

Wang Z, Shen Y, Haapasalo M (2012) Effectiveness of endodontic disinfecting solutions against young and old Enterococcus faecalis biofilms in dentin canals. Journal of Endodontics 38, 1376-9.

Weller RN, Brady JM, Bernier WE (1980) Efficacy of ultrasonic cleaning. Journal of Endodontics 6, 740-3. 

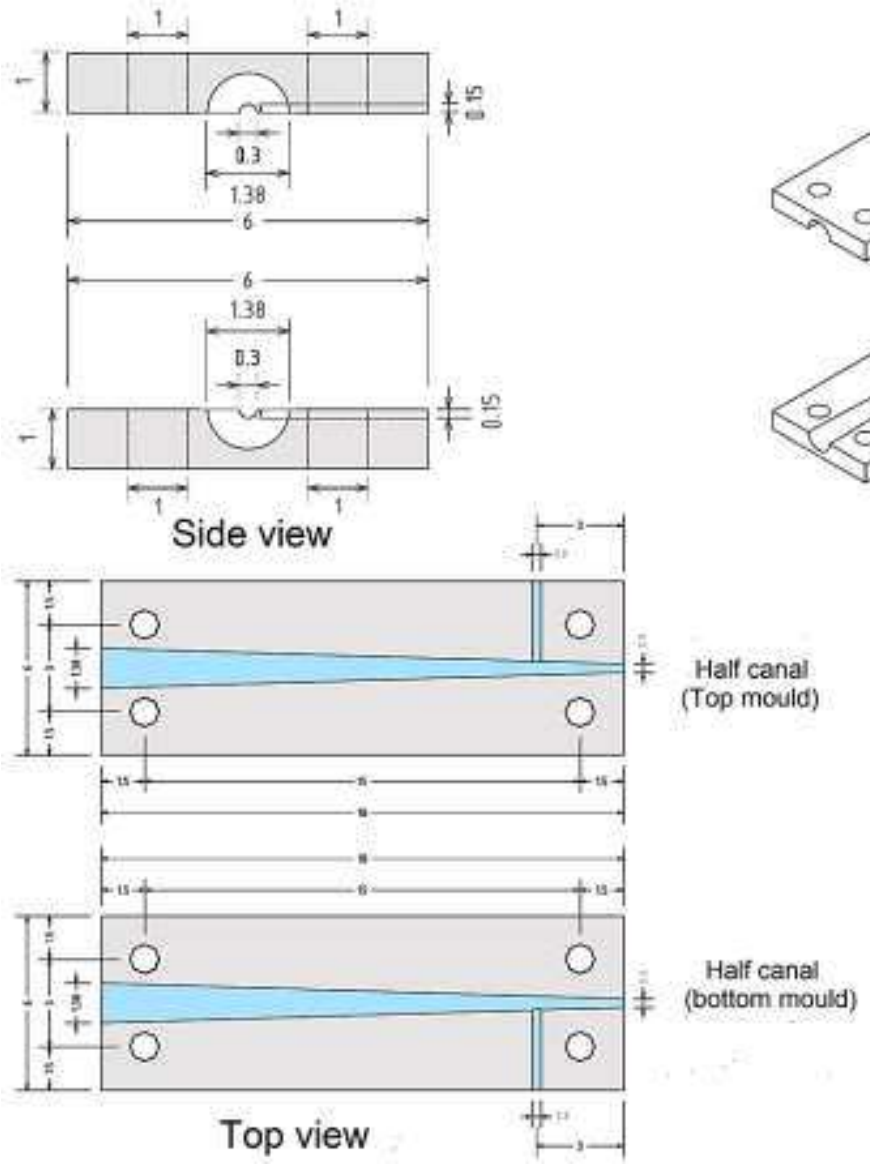
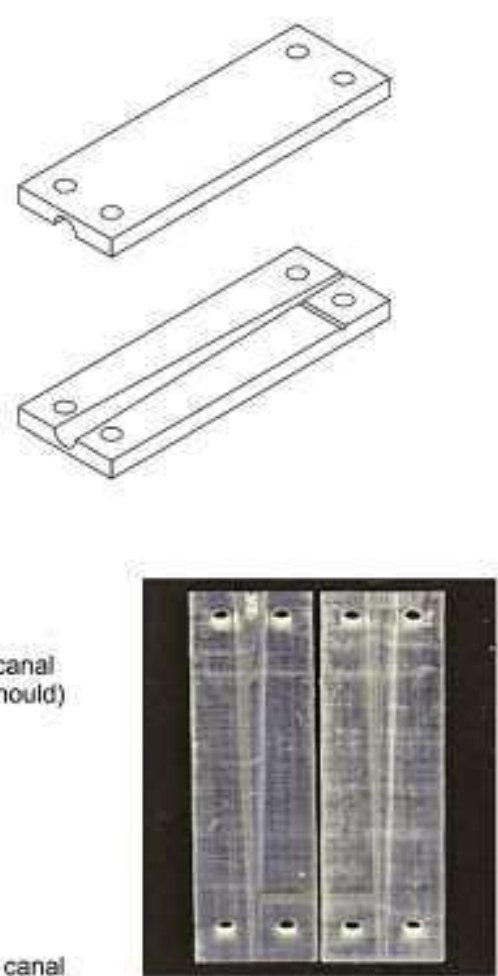

Printed canal model

Figure 1: Image illustrates the design of the complex root canal mode (main and lateral canals. Each half of a simulated canal is of $18 \mathrm{~mm}$ length with $1.38 \mathrm{~mm}$ diameter at the coronal portion and $0.3 \mathrm{~mm}$ diameter at the apical portion. The lower view shows the printed two halves and when they are reassembled, a straight simple canal of 18 $\mathrm{mm}$ length, apical size 30 , and a 0.06 taper is created with lateral canal of $3 \mathrm{~mm}$ length, $0.3 \mathrm{~mm}$ diameter adapted from (Mohmmed et al. 2016) with permission. 


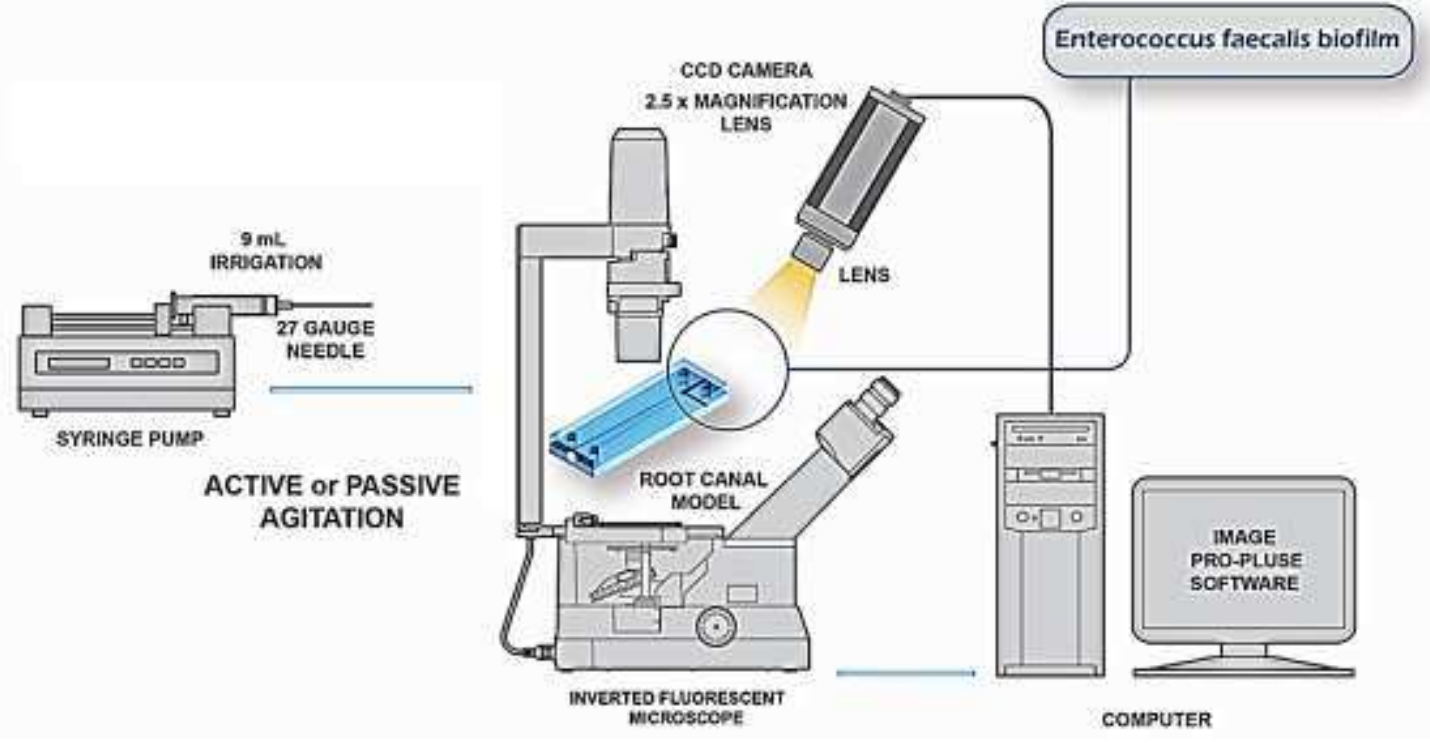

Figure 2: Sketch illustrating the set-up of equipment for recording of the biofilm (biofilm was generated on the apical portion $(3 \mathrm{~mm})$ of the main and lateral $(3 \mathrm{~mm})$ canals model) removal by active or passive $\mathrm{NaOCl}$ irrigation protocol using a camera connected to a $2.5 \times$ lens of an inverted fluorescent microscope. The irrigant was delivered using a syringe with a 27-gauge side-cut open-ended needle, which was attached to a programmable precision syringe pump. The residual biofilm was quantified using computer software (Image-pro Plus 4.5) adapted from (Mohmmed et al. 2016) with permission. 


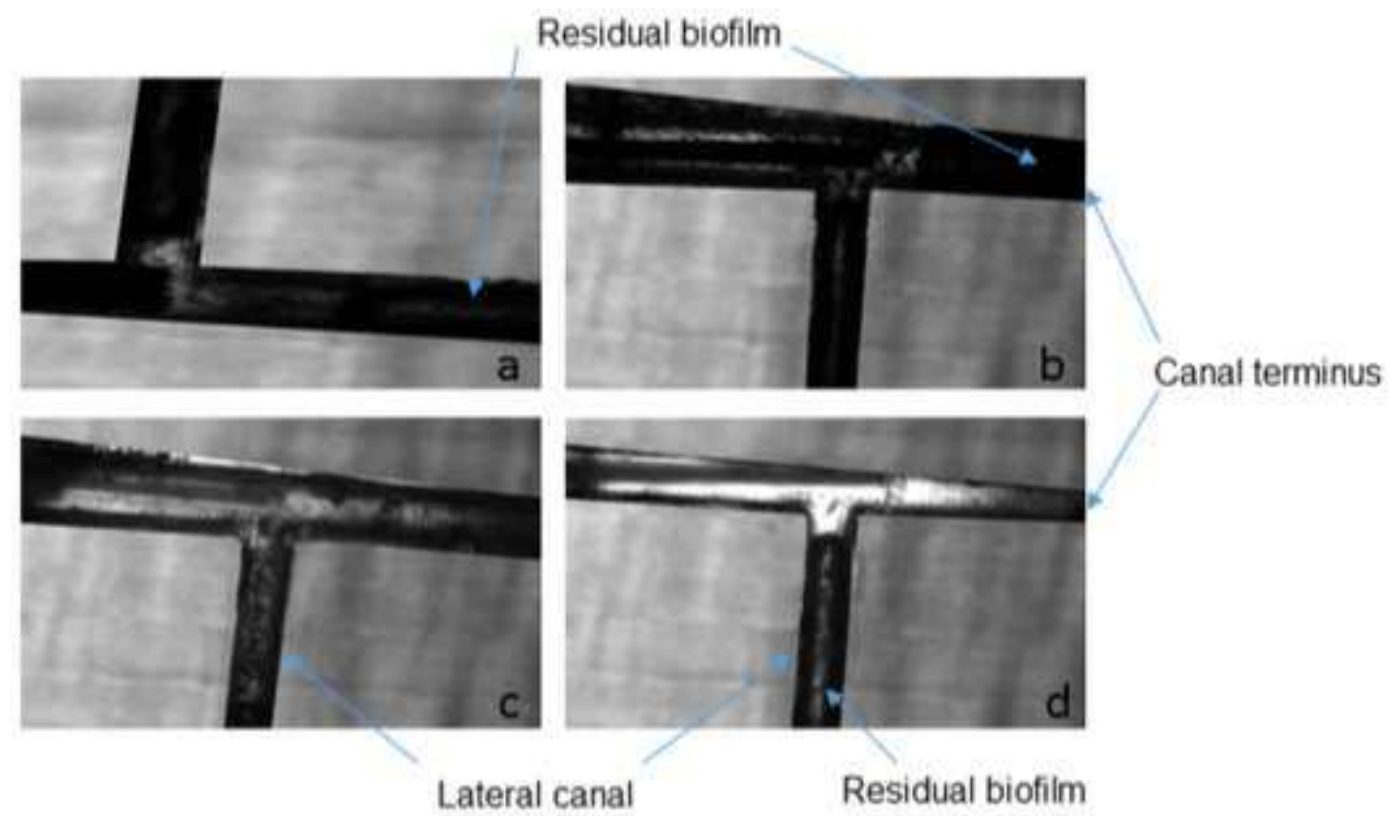

Figure 3: Images illustrate stained E. faecalis biofilm on the canal surface of the root canal model after $\mathrm{NaOCl}$ (a) syringe irrigation, (b) manual agitation, (c) sonic agitation, and (d) ultrasonic agitation protocols. 


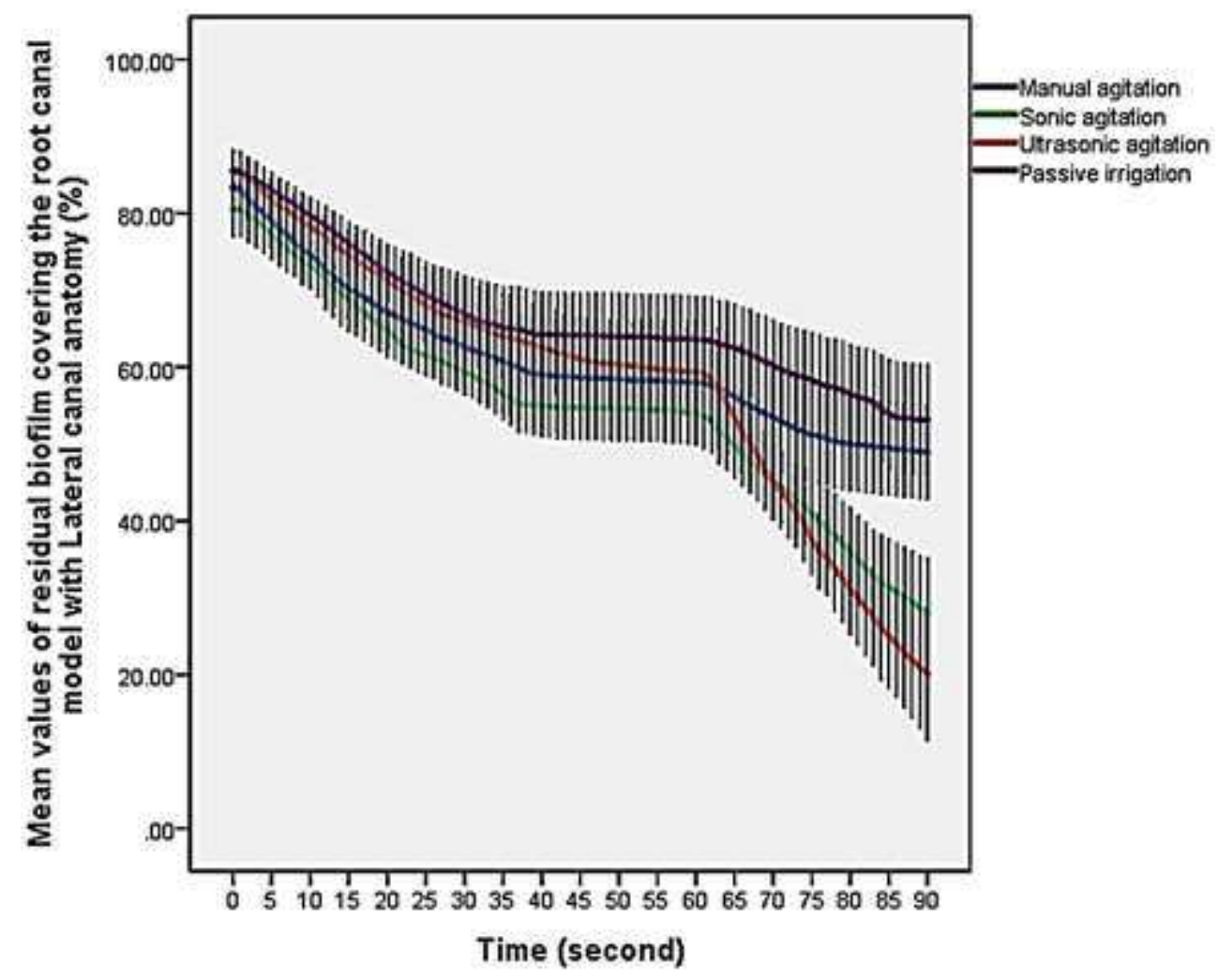

Figure 4: Mean $(95 \% \mathrm{Cl})$ percentages values of the residual biofilm (\%) covering the root lateral canal surface-area over duration (s) of syringe irrigation followed by passive or active irrigation protocols, stratified by type of irrigation ( $n=10$ per group).
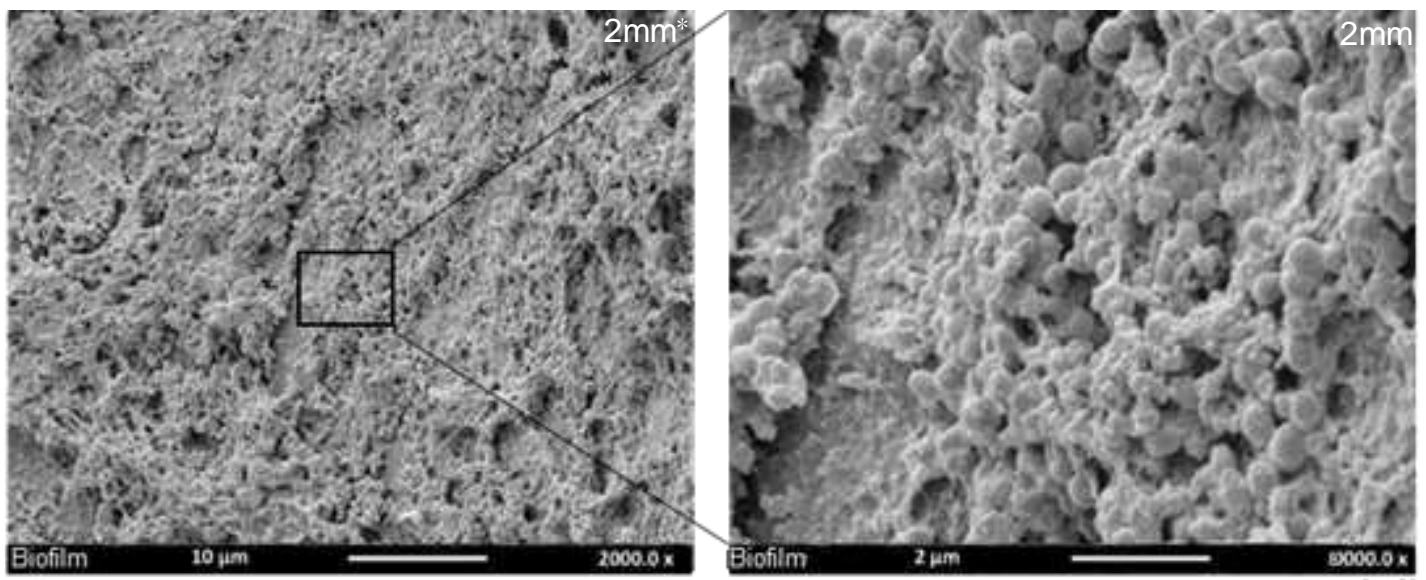

(ai) 
Figure 5: SEM images illustrate (ai) E. faecalis biofilm grown for 10 days. (aii, aiii, and aiv) residual biofilm at $3 \mathrm{~mm}$ from the lateral canal after passive irrigation, manual, sonic protocols respectively. (b) Passive irrigation group; (i) residual biofilm at $2 \mathrm{~mm}$ from the lateral canal terminus; (ii) residual biofilm at $1 \mathrm{~mm}$ from the lateral canal terminus. (c) manual-agitation group; (i) residual biofilm at $2 \mathrm{~mm}$ from the lateral canal terminus; (ii) residual biofilm at $1 \mathrm{~mm}$ from the lateral canal terminus. (d) Sonic agitation group; (i) residual biofilm at $2 \mathrm{~mm}$ from the lateral canal terminus; (ii) residual biofilm at $1 \mathrm{~mm}$ from the lateral canal terminus. (e) Ultrasonic agitation group; (i) residual biofilm at $2 \mathrm{~mm}$ from the lateral canal terminus; (ii) residual biofilm at $1 \mathrm{~mm}$ from the lateral canal terminus. 
Table 1: Generalized linear mixed model analysis to compare the difference in the amount of residual biofilms (\%) covering the lateral canal surface during passive or active irrigation time (the irrigant was either left stagnant in the canal or activated using gutta-percha, sonic or ultrasonic methods for 30 seconds) with $2.5 \% \mathrm{NaOCl}$ irrigant ( $\mathrm{n}=10$ per group).

\begin{tabular}{lccc}
\hline \multicolumn{1}{c}{ Experimental groups } & ${ }^{*}$ Coefficient & 95\% Cl & value \\
\hline Manual agitation vs passive irrigation & 10.78 & $-0.81,22.36$ & 0.068 \\
Sonic agitation vs passive irrigation & 21.04 & $9.46,32.63$ & $\mathbf{0 . 0 0 1}$ \\
Ultrasonic agitation vs passive irrigation & 56.08 & $44.49,67.67$ & $\mathbf{0 . 0 0 1}$ \\
Manual agitation vs ultrasonic agitation & -66.88 & $-78.46,-55.29$ & $\mathbf{0 . 0 1 1}$ \\
Sonic agitation vs ultrasonic agitation & -34.91 & $-46.49,-23.33$ & $\mathbf{0 . 0 1 1}$ \\
Manual agitation vs sonic agitation & -32.31 & $-43.89,-20.72$ & $\mathbf{0 . 0 1 1}$ \\
\hline${ }^{*}$ Coefficient for the residual biofilm, Cl = Confidence interval. & & \\
\hline
\end{tabular}


Table 2: Generalized linear mixed model analysing the effect of time (seconds) on the amount of biofilm removed from the lateral canal surface of each experimental group $(n=10$ per group).

\begin{tabular}{lccc}
\hline \multicolumn{1}{c}{ Experimental groups } & ${ }^{*}$ Coefficient & 95\% Cl & $\begin{array}{c}\boldsymbol{p} \\
\text { value }\end{array}$ \\
\hline Manual agitation vs passive irrigation & -0.06 & $-0.22,0.09$ & 0.428 \\
Sonic agitation vs passive irrigation & -0.51 & $-0.66,-0.36$ & $\mathbf{0 . 0 0 1}$ \\
Ultrasonic agitation vs passive irrigation & -1.01 & $-1.12,-0.85$ & $\mathbf{0 . 0 0 1}$ \\
Manual agitation vs ultrasonic agitation & 0.07 & $0.91,1.22$ & $\mathbf{0 . 0 0 1}$ \\
Sonic agitation vs ultrasonic agitation & 0.49 & $0.34,0.65$ & $\mathbf{0 . 0 0 1}$ \\
Sonic agitation vs manual agitation & 0.58 & $0.43,0.74$ & $\mathbf{0 . 0 0 1}$ \\
\hline${ }^{*}$ Coefficient for time effect represents the rate of biofilm removal, $\mathrm{Cl}=$ Confidence interval.
\end{tabular}

